\title{
Enhanced cuticle accumulation by employing MIXTA-like transcription factors
}

\author{
Yoshimi Oshima, Nobutaka Mitsuda* \\ Bioproduction Research Institute, National Institute of Advanced Industrial Science and Technology (AIST), Central 6, \\ Higashi 1-1-1, Tsukuba, Ibaraki 305-8566, Japan \\ *E-mail: nobutaka.mitsuda@aist.go.jp Tel: +81-29-861-2641 Fax: +81-29-861-3026
}

Received June 9, 2016; accepted June 27, 2016 (Edited by T. Mizoguchi)

\begin{abstract}
The cuticle covers almost the entire aerial surface of terrestrial plants, and provides protection from abiotic and biotic stresses. Cuticles basically consist of wax and cutin, and are produced with variable structures and thicknesses depending on the plant and organ. The application of plant cuticles to improve stress tolerance and wax production requires the deposition of the cuticle at specific times to avoid undesirable side effects. We previously showed that the MYB106 and MYB16 MIXTA-like transcription factors regulate cuticle formation. However, MYB106 over-expression results in severe dwarfism. In this study, we identified genes downstream of these MYB transcription factors and used their promoters to express MYB106 and MYB16 fused to the strong transcriptional activation domain, VP16. Comparisons of plant growth and cuticle morphology revealed that MYB106 and MYB16 preferentially produced cuticles that are typically observed in petals and leaves, respectively. Additionally, the CYP77A6 and CYP86A4 promoters effectively induced cuticle accumulation in leaves and petals, respectively, without inhibiting plant growth. Our strategy may be useful for increasing or altering cuticles in agronomically important plants.
\end{abstract}

Key words: Arabidopsis thaliana, cuticle, nanoridge, transcription factor, wax.

The cuticle of terrestrial plants inhibits organ fusion during development and provides protection from drought, rain, wind, high intensity light, insects, and pathogens (Jeffree 2006). Plants and organs consist of diverse kinds of cuticle that differ in thickness, and their production is induced by developmental or stressresponsive processes (Jeffree 2006). Plants growing in the equatorial region accumulate considerable amounts of wax in the cuticle for enhanced drought tolerance. This type of wax is sometimes harvested by humans for use as natural wax products (Jetter and Kunst 2008). The cuticle covering specific plant tissues forms nanoor micro-ordered structures with a striped pattern called nanoridges. According to a theoretical model, nanoridges form because of mechanical buckling of the cuticle due to differential expansion of epidermal cells and cuticles (Antoniou-Kourounioti et al. 2013). In addition to the above-mentioned cuticle functions, nanoridges influence plant reproduction by regulating the production of structural colors and the "petal effect", which results in the glittering of water drops and formation of ideal structures for insect pollinators. These effects make flowers more attractive to insect pollinators (Feng et al. 2008; Koch et al. 2008; Whitney et al. 2009). Enhancing these functions by increasing or altering the accumulation of cuticle components may help generate plants that are more tolerant to drought, pathogens, and predators, and that produce flowers that are more attractive to humans and pollinators. Despite the importance of the cuticle, the molecular mechanisms underlying the production of different types of cuticle depending on plant species, tissue, and situation have not been fully characterized.

Plant cuticles consist of wax, cutin, and aromatic compounds (Beisson et al. 2012; Jeffree 2006). Mutations in genes encoding proteins associated with cutin biosynthesis, including glycerol-3-phosphate acyltransferase 6 and cytochrome P450 (CYP77A6), which functions with a fatty acid $\omega$-hydroxylase (CYP86A4) to synthesize dihydroxypalmitate, result in cuticle and nanoridge deficiencies (Li-Beisson et al. 2009). In addition to the biosynthesis of cutin and wax monomers, the proper transport of cuticular lipids synthesized in the endoplasmic reticulum is necessary for wax and cutin accumulation outside epidermal cells (Bird et al. 2007; Pighin et al. 2004). An ABC transporter homodimer (ABCG11) and heterodimer (ABCG11ABCG12) are responsible for the export of cutin and wax, respectively (Bird et al. 2007; McFarlane et al. 2010). Additionally, the genes encoding these transporters 
are expressed in epidermal cells (Bird et al. 2007; Luo et al. 2007; Panikashvili et al. 2007; Pighin et al. 2004). The expression of ABCG12, CYP77A6, and CYP86A4 is suppressed in loss-of-function lines, but induced in gain-of-function lines involving the MYB106/NOECK MIXTA-like transcription factor (TF), which regulates cuticle production (Oshima et al. 2013a).

The MIXTA-like TFs were originally reported to regulate epidermal cell outgrowth in petal conical cells and trichomes (Baumann et al. 2007; Folkers et al. 1997; Glover et al. 1998; Jaffé et al. 2007; Jakoby et al. 2008; Noda et al. 1994). We previously revealed that MYB106 and MYB16, which is the closest MYB106 paralog, regulate cuticle development in Arabidopsis thaliana and Torenia fournieri (Oshima et al. 2013a; Oshima and Mitsuda 2013b). In vegetative organs, the null mutation of MYB106 does not lead to cuticular deficiencies except in trichomes. However, the double knockout/down of MYB106 and MYB16 results in an apparent leaf cuticle functional deficiency (Oshima and Mitsuda 2013b). Using these mutant lines, we showed that MYB106 and MYB16 are required for the formation of cuticle nanoridges in petals and stamens. Furthermore, we reported that MYB106 is sufficient for the ectopic production of nanoridges (Oshima et al. 2013a). However, plants over-expressing MYB106 exhibit severe dwarfism and are not suitable for commercial production.

In this study, we generated transgenic plants with a greater abundance of cuticle than the wild-type controls. The transgenic plants exhibited fewer growth defects because of the promoters of genes downstream of MIXTA-like TFs. Analyses of cuticle morphology indicated that MIXTA-like TFs induced the production of different types of cuticle, which are usually only observed in specific tissues.

\section{Materials and methods}

\section{Plant materials and growth conditions}

Arabidopsis thaliana ecotype Columbia- 0 was used as the plant material in this study. The 35S:MYB106:HSP A. thaliana line was produced as previously described (Oshima et al. 2013a). Seedlings were grown on solid Murashige and Skoog medium and then transferred to soil approximately 3 weeks after germination. Plants were grown at $22^{\circ} \mathrm{C}$ using a 16 -h light/8-h dark photoperiod.

\section{Transient expression assay}

The transient reporter-effector particle bombardment assay was conducted as previously described (Mitsuda et al. 2011). To produce the reporter constructs, DNA regions approximately 1,000 bp upstream from CYP77A6 and about 2,000 bp upstream from $A B C G 12$ were amplified using a polymerase chain reaction with the following primers: CYP77A6proF 5'-
GGG GAC AAC TTT GTA TAG AAA AGT TGT TAT CTT CCC GGA ATT AGT GAA GACCC-3', CYP77A6proR 5'-GGG GAC TGC TTT TTT GTA CAA ACT TGG CAT TTT TAG CTT CTT GTT TTT CTT CTT-3', ABCG12proF 5'-GGG GAC AAC TTT GTA TAG AAA AGT TGT TTT TCT GGG GTT TTT GTA GGG TTT GG-3', and ABCG12proR 5'-GGG GAC TGC TTT TTT GTA CAA ACT TGG CAT TGT TTT TGT TTG ATC TTG AAA AAG- ${ }^{\prime}$. The amplicons were cloned into the pDONRG_P4P1R vector (Oshima et al., 2011) using the Gateway BP reaction. The contents of each plasmid were inserted into the R4L1pDEST LUC_HSP vector (Oshima et al. 2013a) using the Gateway LR reaction. The pDEST35S_MYB106_VP16 and pDEST35S_ MYB16_VP16 plasmids used as effectors and the pDEST35S_ VAMP722 vector used as the negative control have been previously described (Oshima et al. 2013a). Rosette leaves from A. thaliana plants grown under short-day conditions (i.e., 10-h light/14-h dark cycle) were bombarded with the effector and reporter plasmids. As an internal reference, rosette leaves were bombarded with pRLHSP or phRLHSP (Oshima et al. 2013a) to normalize the reporter activities.

\section{Plasmid construction and plant transformation}

The DNA regions upstream from CYP77A6, ABCG12, and CYP86A4 described above and in a published report (Oshima et al. 2013a) were used to generate the following constructs: CYP86A4pro:MYB106-VP16, CYP86A4pro:MYB16-VP16, CYP77A6pro:MYB106-VP16, CYP77A6pro:MYB16-VP16, ABCG12pro:MYB106-VP16, and ABCG12pro:MYB16-VP16. The cloned promoter fragments and MYB106 or MYB16 coding regions in the conventional entry clone were transferred using the multisite Gateway LR reaction into the R4pGWB5_VP16_ HSP T-DNA vector, which is based on the R4pGWB5_SRDX_ HSP vector (Oshima et al. 2011). The SRDX of R4pGWB5_ SRDX_HSP was replaced with the herpes simplex virus VP16 to produce the R4pGWB5_VP16_HSP vector. The above-listed constructs were inserted into $A$. thaliana plants using a floral dip method (Clough and Bent 1998).

\section{Scanning electron microscopy}

Fresh plant samples were examined using a Real 3D system scanning electron microscope (models VE8800; Keyence) at an accelerating voltage of 1 or $1.5 \mathrm{kV}$.

\section{Results and discussion}

\section{Constitutive expression of MIXTA-like transcription factors}

We previously analyzed transgenic plants constitutively expressing MYB106 or MYB106-VP16 under the control of the CaMV $35 S$ promoter. We showed that MYB106 is able to induce the over-accumulation of cuticle components and ectopic formation of nanoridges in leaves and carpels (Oshima et al. 2013a). However, the transgenic plants were much smaller than the wildtype controls (Figure 1), and the induction of cuticle 


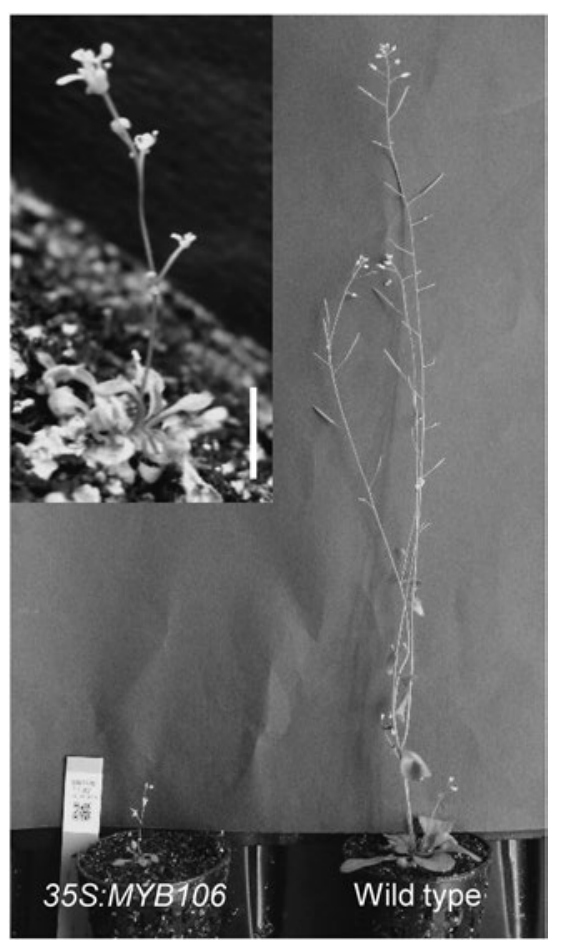

Figure 1. Phenotype of the 35S:MYB106 plant. A 6-week-old 35S:MYB106 transgenic Arabidopsis thaliana plant and a wildtype control are presented. The inset provides a close-up view of a 35S:MYB106 plant. Bar represents $5 \mathrm{~mm}$.

production by the TF was difficult to evaluate. In these plants, the ectopic accumulation of a thick cuticle may have prevented cellular growth, or the side effects may have led to decreased plant growth. The inhibited growth caused by the overexpression of TF genes regulating cuticle formation was also observed in studies involving WAX INDUCER 1 (WIN1)/SHINE1 (SHN1) and Eutrema salsugineum WAX1 (EsWAX1), which is an ortholog of the MYB96 stress-inducible cuticle regulator (Broun et al. 2004; Zhu et al. 2014). However, the use of the stressinducible $R D 29 \mathrm{~A}$ promoter and a chemical-inducible promoter to express EsWAX1 and WIN1/SHN1, respectively, improves drought tolerance (with no adverse effects on growth) through increased accumulation of cuticular wax and other effects not directly related to wax accumulation (Yang et al. 2011; Zhu et al. 2014).

\section{Regulation of promoter activity related to cuticle formation}

To produce healthy plants with a thick cuticle, the genes influencing cuticle production should be under the control of a promoter that is active during specific periods. We hypothesized that the promoters of genes downstream of the cascade regulating cuticle formation should ideally control the expression of TF genes influencing cuticle formation. This would ensure the promoters are active at appropriate times, but would also help boost promoter activity through a positive feedback
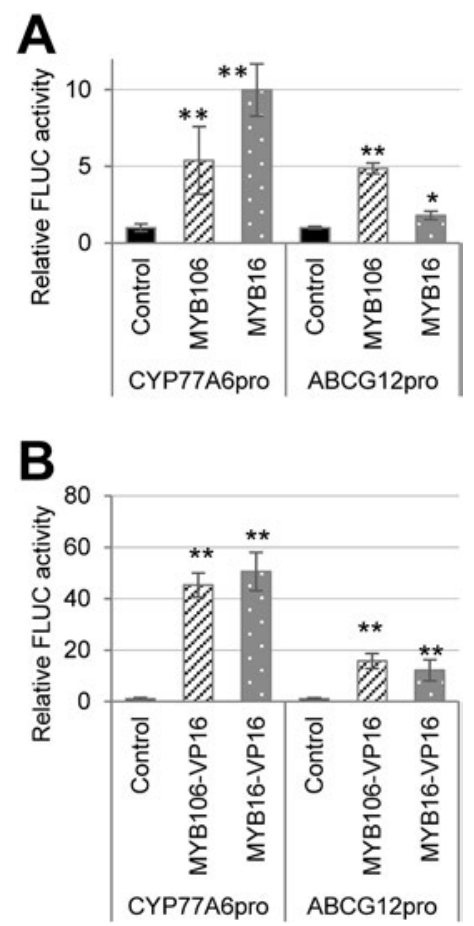

Figure 2. Reporter-effector assay involving MIXTA-like transcription factors and cuticle-related gene promoters. The $L U C$ reporter gene under the control of the CYP77A6 and ABCG12 promoters was transiently expressed along with VAMP722-VP16 (control), MYB106, and MYB16 alone (A) or fused to VP16 (B) as the effector. The LUC activity level when the control was co-expressed was set to 1 . Error bars represent standard errors $(n=5$ or 6$)$. Single and double asterisks indicate $p<0.05$ and $p<0.01$ significance levels, respectively, according to Welch's $t$ test.

loop (Yang et al. 2013). MYB106 functions during epidermal cell maturation, as indicated by the fact the trichomes of myb106 mutants remain morphologically immature (Gilding and Marks 2010; Oshima et al. 2013a). Therefore, the genes downstream of MYB106 are likely expressed during cell maturation processes associated with cuticle thickening. We previously identified putative genes downstream of MYB106 by analyzing gene expression levels in plants carrying 35S:MYB106-SRDX and 35S:MYB106-VP16 (Oshima et al. 2013a). We confirmed the CYP86A4 promoter is activated by MYB106 and MYB16 using the reportereffector assay (Oshima et al. 2013a). In the current study, we further examined the relationship between promoters and TFs, and observed that MYB106 and MYB16 activate the CYP77A6 and ABCG12 promoters (Figure 2A). MYB106 activated CYP86A4 promoter much more than CYP77A6 and ABCG12 promoters whereas MYB16 similarly activated three promoters (Oshima et al. 2013a). CYP77A6 and ABCG12 promoters may have lower affinity with MYB106 or be regulated by different activation mechanism from CYP86A4 promoter. Additionally, a fusion protein consisting of these MYB proteins and VP16 activated CYP77A6 and ABCG12 promoters more effectively than the MYB proteins 


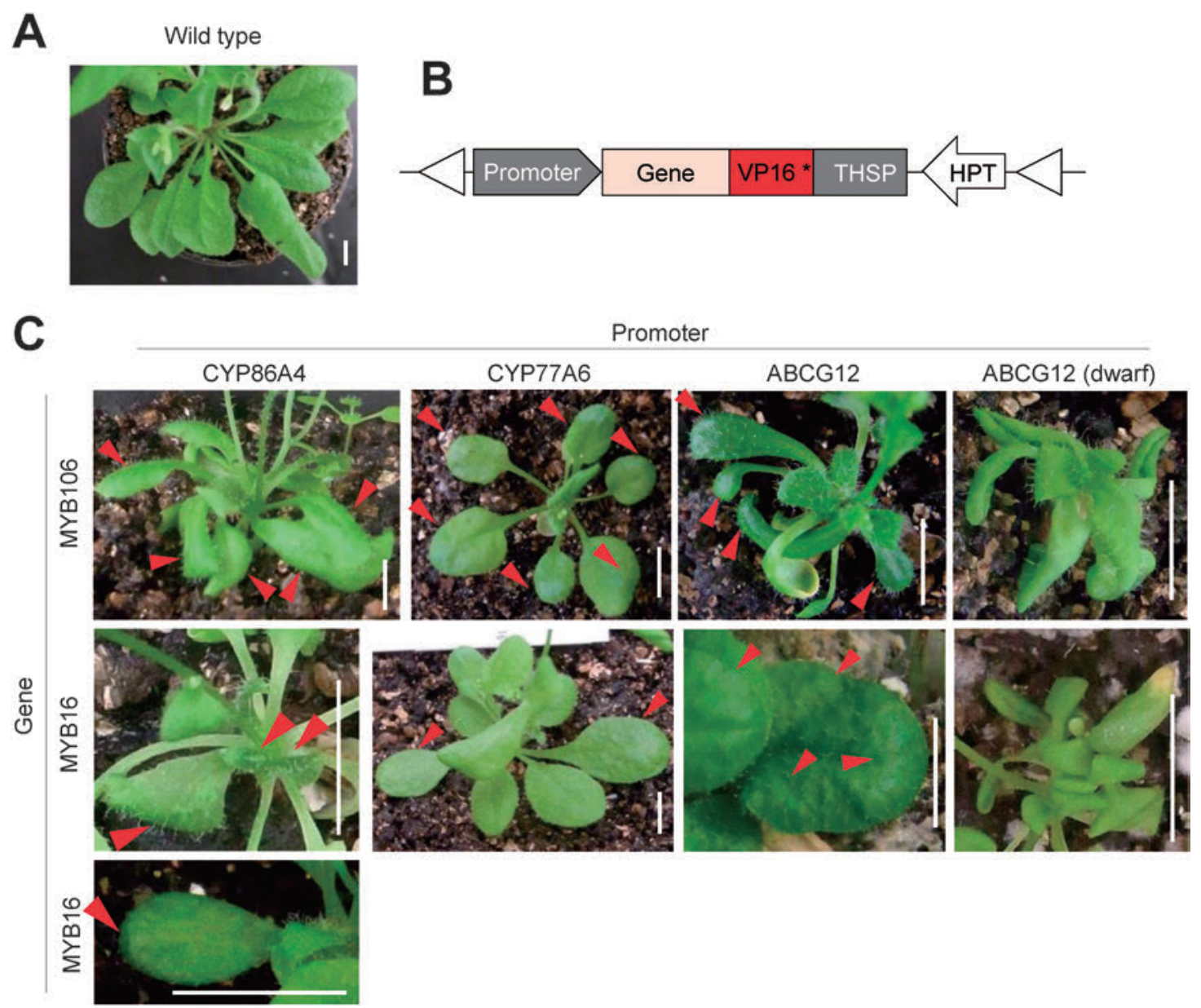

Figure 3. Phenotype of leaves from plants expressing MYB106-VP16 or MYB16-VP16 under the control of the CYP86A4, CYP77A6, or ABCG12 promoters. (A) Rosette leaves of a wild-type plant. (B) Schematic representation of the construct used to express MIXTA-like MYB genes. The promoter and gene were inserted into vectors using the combinations presented in $(C)$. Open triangles indicate the right and left border sequences. HSP, Arabidopsis thaliana HSP18.2 terminator; HPT, hygromycin B phosphotransferase. (C) Phenotype of transgenic plants expressing MYB106-VP16 or MYB16-VP16 under the control of the CYP86A4, CYP77A6, or ABCG12 promoters. Plants produced shiny leaves (red arrow heads) or exhibited dwarfism. Bars $=5 \mathrm{~mm}$.

alone (Figure 2B). These results suggest that CYP77A6 and $A B C G 12$ as well as CYP86A4 are common targets of MIXTA-like MYB TFs.

\section{Effects of the CYP77A6, CYP86A4, and ABCG12 promoters on leaf phenotypes}

We expressed MYB106 and MYB16 fused to VP16 under the control of the CYP77A6, CYP86A4, and ABCG12 promoters to generate plants with a thick cuticle. We analyzed the growth and cuticle types of the transgenic plants. All six combinations of TF and promoter induced the production of shiny leaves, suggesting an excessive accumulation of cuticle components (Figure 3). Generally, the smaller plants were shinier. Additionally, the $A B C G 12$ promoter induced the production of small and shiny leaves, and also led to dwarfism and the generation of pale green epinastic leaves (Figure 3C). This growth defect was similar to or stronger than that observed in plants expressing MYB106 and MYB16 under the control of the CaMV 35S promoter (Figure 1). The CYP86A4pro:MYB106-VP16 and CYP86A4pro:MYB16-VP16 plants also generated epinastic leaves (Figure 3C). In contrast, the shapes of leaves in plants carrying the CYP77A6 promoter were normal, suggesting this promoter did not adversely affect plant growth during the vegetative stage (Figure 3C).

\section{Cuticle accumulation on leaves}

We analyzed the cuticle of shiny leaves from six transgenic lines using a scanning electron microscope. The cuticle of wild-type leaves was smooth with a very faint spot or brush mark-like pattern, and its surface became rough with a slightly indistinct cell margin when the leaves aged (i.e., smooth cuticle; Figure 4G-I). In the MYB106-VP16 lines, smooth cuticles and ectopic nanoridges accumulated on leaves (Figure 4A-C). Additionally, MYB16-VP16 induced the accumulation of epicuticular wax crystals (Figure 4D) and a smooth cuticle with a marble pattern. There were also more mass spots compared with wild-type leaves 


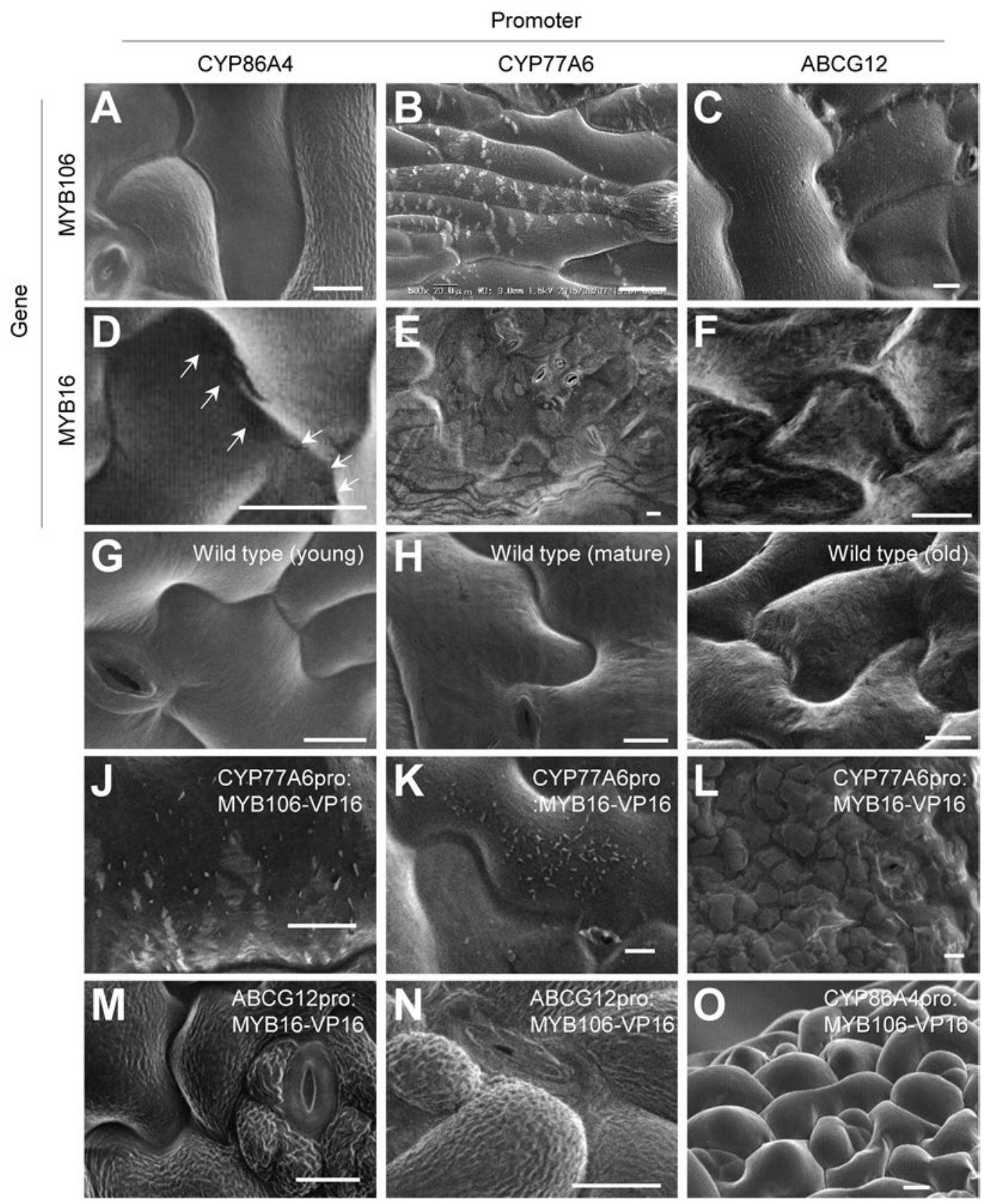

Figure 4. Surface of leaves from plants expressing MYB106-VP16 or MYB16-VP16 under the control of the CYP86A4, CYP77A6, or ABCG12 promoters. Surface of rosette leaves observed using a scanning electron microscope. (A-F) Surface of shiny rosette leaves of transgenic plants expressing MYB106-VP16 or MYB16-VP16 under the control of the CYP86A4, CYP77A6, or ABCG12 promoters. Arrows indicate epicuticular wax crystals (D). (G-I) Surface of wild-type rosette leaves. (J-L) Surface of shiny rosette leaves from plants expressing MYB106-VP16 (J) or MYB16-VP16 $(\mathrm{K}, \mathrm{L})$ under the control of the CYP77A6 promoter. (M, N) Surface of leaves from a dwarf plant expressing MYB16-VP16 (M) or MYB106-VP16 (N) under the control of the ABCG12 promoter. (O) Surface of leaves of a CYP86A4pro:MYB106-VP16 plant. Bars $=10 \mu \mathrm{m}$.

(Figure 4E, F). Unlike in wild-type leaves, which had clear cell margins (Figure 4G-I), cuticle components accumulated in the cell margins of transgenic leaves (Figure 4A-C, E, F). Cellular outgrowth was observed in lines carrying CYP86A4pro:MYB16-VP16, suggesting epinastic leaves were the result of morphological changes to epidermal cells (Figure 4O). There was a greater abundance of cuticle in CYP77A6pro:MYB16VP16 plants than in MYB106-VP16 lines, and the cell margins were completely filled with cuticle (Figure $4 \mathrm{E}$ ).
We also observed the occasional crack in the cuticle of CYP77A6pro:MYB16-VP16 plants (Figure 4L). In most cases, these cracks appeared to be filled with newly secreted cuticle, providing further evidence of the overaccumulation of cuticle. Furthermore, the cracks may have prevented the generation of severely epinastic leaves similar to those of CYP86A4pro:MYB16-VP16 plants. We also sometimes observed the accumulation of wax crystals in MYB106-VP16 and MYB16-VP16 lines (Figure 4D, J, K). In contrast, the pale green dwarf 
lines carrying the $A B C G 12$ promoter accumulated a high density of nanoridges (Figure $4 \mathrm{M}, \mathrm{N}$ ). Thus, the accumulation of the smooth cuticle was believed to be responsible for the production of shiny leaves following the expression of MIXTA-like TF genes. These data suggest that MYB106 and MYB16 predominantly induce nanoridge formation and the accumulation of leaf-type cuticles, respectively, although they have overlapping roles in wax crystal production, cuticle accumulation, and nanoridge formation. Finally, the CYP77A6 promoter appeared to induce the greatest production of cuticle on leaves.

\section{Cuticle accumulation on floral organs}

To analyze flowers, we focused on the lines carrying the CYP86A4 and CYP77A6 promoters because the ABCG12 promoter induced dwarfism. The expression of MYB106VP16 and MYB16-VP16 under the control of the CYP86A4 promoter commonly induced the production of matte-white and short petals that glittered less than the wild-type petals (Figure 5A-E). The petals of CYP86A4pro:MYB106-VP16 and CYP86A4pro:MYB16$V P 16$ plants had a hard and thick texture whereas wildtype petals were soft (Figure 5A-C). Scanning electron microscopy analysis revealed an increased density of nanoridges on the adaxial surface of the petal epidermis, which exhibited cellular outgrowth (Figure 5F-H). The CYP77A6 promoter induced similar phenotypes regarding the induction of outgrowth and short petals, but did not increase nanoridge density (Figure 5I, J) or produce matte-white petals as much as the CYP86A4 promoter (Figure 5B-E, K, L). An increase in nanoridge density and ectopic outgrowth was also observed in other nanoridge-forming cells on the abaxial side of petals and in stamens and sepals. However, the accumulation of epicuticular wax crystals or leaf-type smooth cuticles was not induced by MIXTA-like MYBs in these tissues (Figure 6). These results suggest that the CYP86A4 promoter induces increase of cuticle nanoridges and alters the resultant petal texture more effectively than the CYP77A6 promoter in floral organs, and that MIXTAlike TFs consistently induce nanoridge production in nanoridge-forming cells. Our observations regarding the petal adaxial surface are consistent with those of plants expressing MYB106-VP16 under the control of the petalspecific InMYB1_1kb promoter (Azuma et al. 2016).

\section{Expression patterns of CYP86A4, CYP77A6, and ABCG12}

The activity levels of the CYP86A4, CYP77A6, and $A B C G 12$ promoters may be predicted based on the respective gene expression patterns. According to a publicly available microarray database, CYP86A4 and CYP77A6 have similar expression patterns, and are highly expressed in growing floral organs, especially
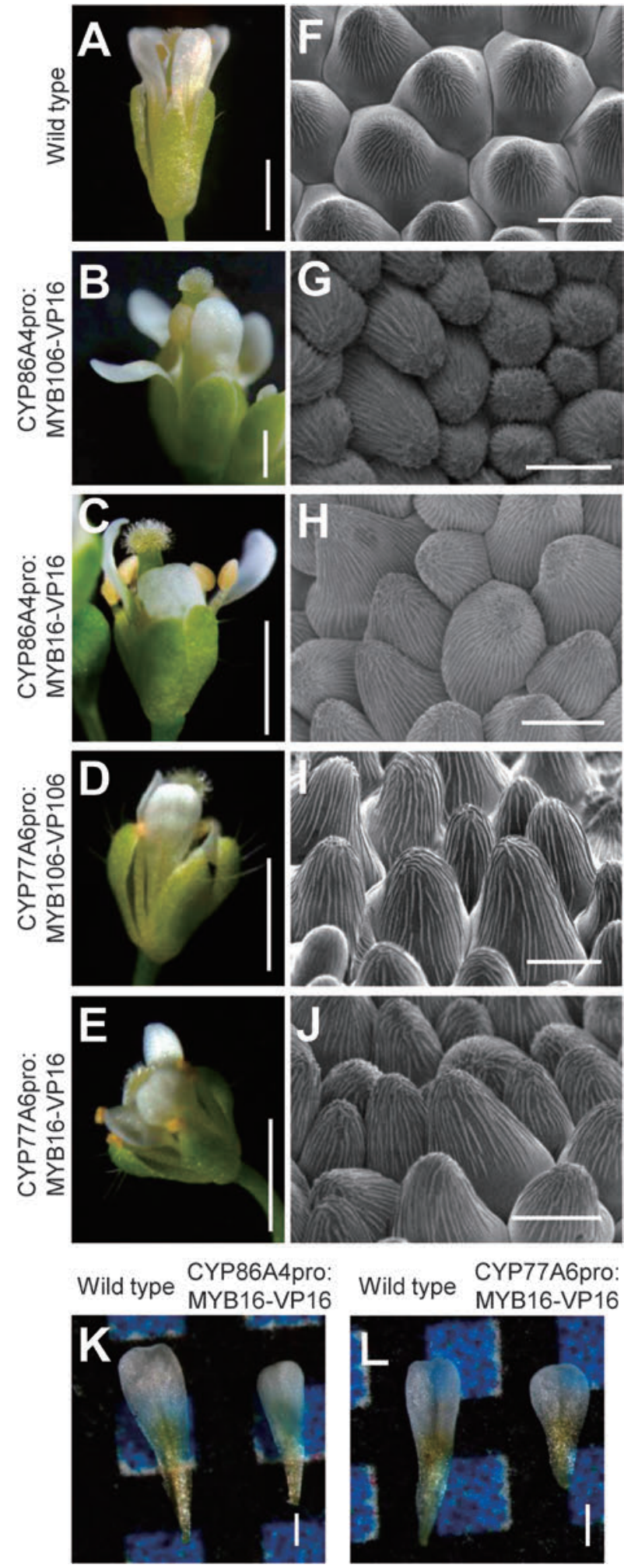

Wild type CYP77A6pro:

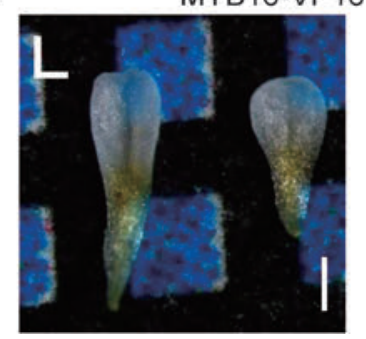

Figure 5. Phenotype of petals from plants expressing MYB106VP16 or MYB16-VP16 under the control of the CYP86A4 or CYP77A6 promoters. Petals (A-E) and the adaxial surface of petals observed using a scanning electron microscope (F-J) in wild-type plants $(\mathrm{A}, \mathrm{F})$ and transgenic plants expressing MYB106-VP16 (B, D, G, I) or MYB16VP16 (C, H, E, J) under the control of the CYP86A4 (B, C, G, H) or CYP77A6 (D, E, I, J) promoters. (K, L) Comparisons of petal color of CYP86A4pro:MYB16-VP16 (K) and CYP77A6pro:MYB16-VP16 (L) with wild type. Bars $=0.5 \mathrm{~mm}$ (left column) and $10 \mu \mathrm{m}$ (right column). 

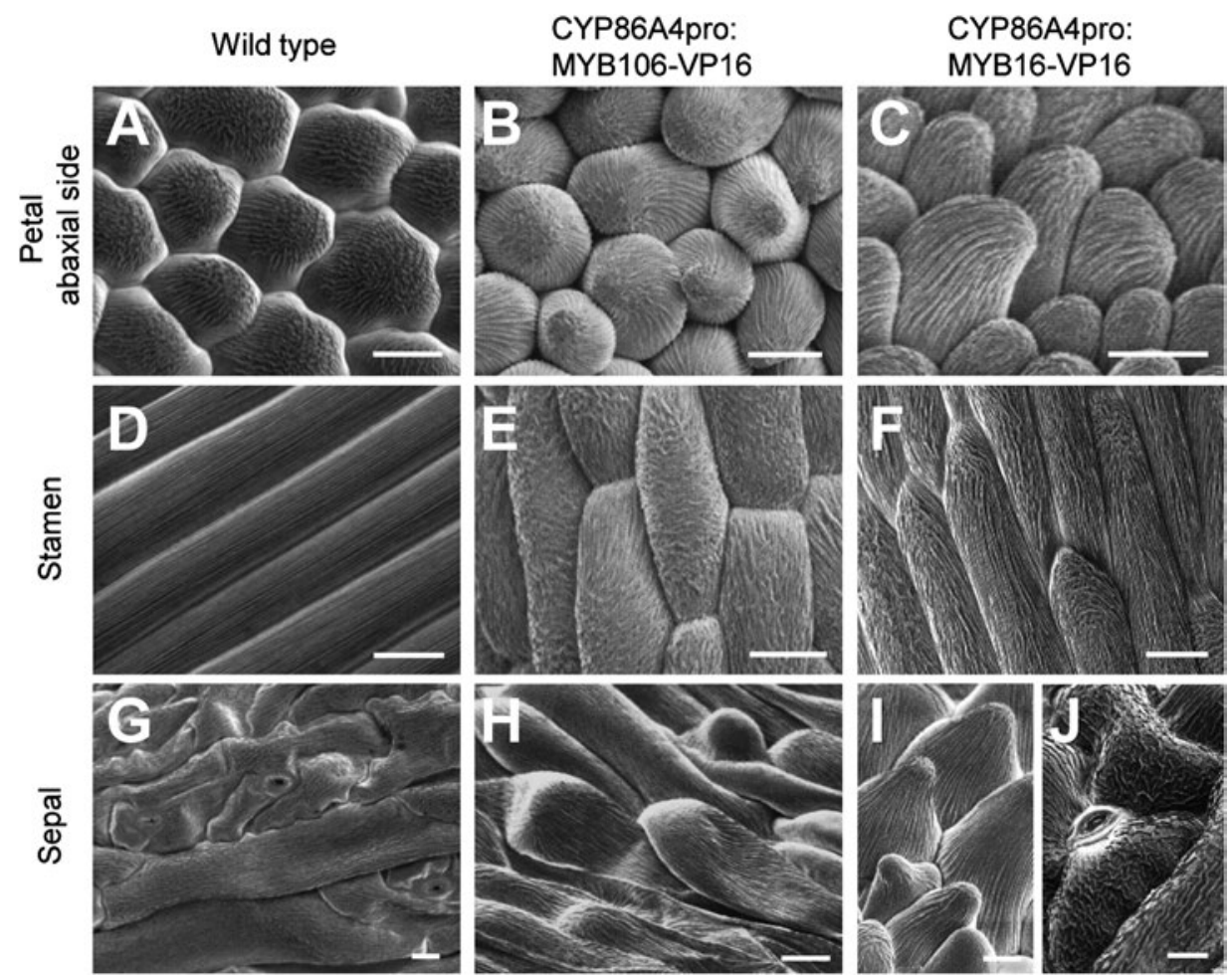

Figure 6. Surface of floral organs from plants expressing MYB106-VP16 or MYB16-VP16 under the control of the CYP86A4 promoter. The surface of the abaxial side of a petal (A-C) as well as a stamen (D-F) and sepal (G-J) observed using a scanning electron microscope in wild-type (A, D, G), CYP86A4pro:MYB106-VP16 (B, E, H), and CYP86A4pro:MYB16-VP16 (C, F, I, J) plants. Images of two positions on a sepal from a CYP86A4pro:MYB16-VP16 plant are presented (I, J). Bars $=10 \mu \mathrm{m}$.

the petals (Supplementary Figure 1; Aoki et al. 2016; Winter et al. 2007). Duan and Schuler (2005) reported that CYP86A4 is expressed most highly in flowers, followed by stems and siliques, while its expression level is low in seedlings and older rosette leaves. These data are consistent with our results indicating CYP86A4 and CYP77A6 promoters induced the highest cuticle accumulation in floral organs, and moderate levels in leaves. The differences between CYP86A4 and CYP77A6 promoters regarding cuticle accumulation and plant growth may be due to variabilities in the efficiency of the positive feedback loop involving the MIXTA-like MYBs and promoters rather than the organ specificity of each promoter. The expression pattern of MYB106 and MYB16 is similar to that of CYP86A4 and CYP77A6 (Supplementary Figure 1C). This suggests that the use of CYP86A4 and CYP77A6 promoter induced the expression of MYB106 and MYB16 similarly to their own expression, resulting in less growth defect. In contrast, according to data available in a microarray database, ABCG12 is expressed in all stages and tissues during plant development (Supplementary Figure 1A; Winter et al 2007). This is not correlated with the expression of MYB106 and MYB16 (Oshima et al. 2013a; Supplementary Figure 1C). Therefore, the ABCG12 promoter is likely responsible for inhibiting growth, similar to the CaMV $35 S$ promoter.

\section{Conclusion}

In this study, we generated transgenic plants expressing MYB106-VP16 or MYB16-VP16 under the control of the CYP77A6 and CYP86A4 promoters to ensure uninhibited growth. We revealed that MYB106 and MYB16 preferentially induce the production of nanoridges (which are usually only observed in floral organs) and leaf-type cuticles, respectively, in vegetative organs. They also commonly increase the abundance of nanoridges in floral organs. Additionally, we established that CYP77A6 and CYP86A4 promoters are suitable for expressing genes in vegetative and floral organs, respectively. Our findings provide important insights related to cuticle accumulation, molecular mechanisms regulating cuticle formation, and biological functions of various cuticles.

\section{Acknowledgements}

The authors thank Y. Sugimoto, M. Yamada, A. Kuwazawa, Y. Takiguchi, and F. Tobe for their technical assistance. This work was partly supported by a Grant-in Aid for Young Scientists (B) from the Japan Society for the Promotion of Science to YO (JP26840103).

\section{References}

Antoniou Kourounioti RL, Band LR, Fozard JA, Hampstead A, Lovrics A, Moyroud E, Vignolini S, King JR, Jensen OE, Glover 
BJ (2013) Buckling as an origin of ordered cuticular patterns in flower petals. J R Soc Interface 10: 20120847

Aoki Y, Okamura Y, Tadaka S, Kinoshita K, Obayashi T (2016) ATTED-II in 2016: A plant coexpression database towards lineage-specific coexpression. Plant Cell Physiol 57: e5

Azuma M, Morimoto R, Hirose M, Morita Y, Hoshino A, Iida S, Oshima Y, Mitsuda N, Ohme-Takagi M, Shiratake K (2016) A petal-specific inMYB1 promoter from japanese morning glory: A useful tool for molecular breeding of floricultural crops. Plant Biotechnol J 14: 354-363

Baumann K, Perez-Rodriguez M, Bradley D, Venail J, Bailey P, Jin H, Koes R, Roberts K, Martin C (2007) Control of cell and petal morphogenesis by R2R3 MYB transcription factors. Development 134: 1691-1701

Beisson F, Li-Beisson Y, Pollard M (2012) Solving the puzzles of cutin and suberin polymer biosynthesis. Curr Opin Plant Biol 15: 329-337

Bird D, Beisson F, Brigham A, Shin J, Greer S, Jetter R, Kunst L, Wu X, Yephremov A, Samuels L (2007) Characterization of Arabidopsis ABCG11/WBC11, an ATP binding cassette (ABC) transporter that is required for cuticular lipid secretion. Plant $J$ 52: 485-498

Broun P, Poindexter P, Osborne E, Jiang CZ, Riechmann JL (2004) WIN1, a transcriptional activator of epidermal wax accumulation in Arabidopsis. Proc Natl Acad Sci USA 101: 4706-4711

Clough SJ, Bent AF (1998) Floral dip: A simplified method for Agrobacterium-mediated transformation of Arabidopsis thaliana. Plant J 16: 735-743

Duan H, Schuler MA (2005) Differential expression and evolution of the Arabidopsis CYP86A subfamily. Plant Physiol 137: 1067-1081

Feng L, Zhang Y, Xi J, Zhu Y, Wang N, Xia F, Jiang L (2008) Petal effect: A superhydrophobic state with high adhesive force. Langmuir 24: 4114-4119

Folkers U, Berger J, Hulskamp M (1997) Cell morphogenesis of trichomes in Arabidopsis: Differential control of primary and secondary branching by branch initiation regulators and cell growth. Development 124: 3779-3786

Gilding EK, Marks MD (2010) Analysis of purified glabra3shapeshifter trichomes reveals a role for NOECK in regulating early trichome morphogenic events. Plant J 64: 304-317

Glover BJ, Perez-Rodriguez M, Martin C (1998) Development of several epidermal cell types can be specified by the same MYBrelated plant transcription factor. Development 125: 3497-3508

Jaffé FW, Tattersall A, Glover BJ (2007) A truncated MYB transcription factor from antirrhinum majus regulates epidermal cell outgrowth. J Exp Bot 58: 1515-1524

Jakoby MJ, Falkenhan D, Mader MT, Brininstool G, Wischnitzki E, Platz N, Hudson A, Hulskamp M, Larkin J, Schnittger A (2008) Transcriptional profiling of mature Arabidopsis trichomes reveals that NOECK encodes the MIXTA-like transcriptional regulator MYB106. Plant Physiol 148: 1583-1602

Jeffree CE (2006) The fine structure of the plant cuticle. In: Riederer M, Müller C (eds) Biology of the plant cuticle, Annual Plant Reviews, Volume 23. Blackwell Publishing, Oxford, pp11-125

Jetter R, Kunst L (2008) Plant surface lipid biosynthetic pathways and their utility for metabolic engineering of waxes and hydrocarbon biofuels. Plant J 54: 670-683
Koch K, Bhushan B, Barthlott W (2008) Diversity of structure, morphology and wetting of plant surfaces. Soft Matter 4: 1943-1963

Li-Beisson Y, Pollard M, Sauveplane V, Pinot F, Ohlrogge J, Beisson F (2009) Nanoridges that characterize the surface morphology of flowers require the synthesis of cutin polyester. Proc Natl Acad Sci USA 106: 22008-22013

Luo B, Xue XY, Hu WL, Wang LJ, Chen XY (2007) An ABC transporter gene of Arabidopsis thaliana, AtWBC11, is involved in cuticle development and prevention of organ fusion. Plant Cell Physiol 48: 1790-1802

McFarlane HE, Shin JJ, Bird DA, Samuels AL (2010) Arabidopsis ABCG transporters, which are required for export of diverse cuticular lipids, dimerize in different combinations. Plant Cell 22: 3066-3075

Mitsuda N, Matsui K, Ikeda M, Nakata M, Oshima Y, Nagatoshi Y, Ohme-Takagi M (2011) CRES-T, an effective gene silencing system utilizing chimeric repressors. Methods Mol Biol 754: $87-105$

Noda K, Glover BJ, Linstead P, Martin C (1994) Flower colour intensity depends on specialized cell shape controlled by a mybrelated transcription factor. Nature 369: 661-664

Oshima Y, Mitsuda N (2013b) The MIXTA-like Transcription factor MYB16 is a major regulator of cuticle formation in vegetative organs. Plant Signal Behav 8: e26826

Oshima Y, Mitsuda N, Nakata M, Nakagawa T, Nagaya S, Kato K, Ohme-Takagi M (2011) Novel vector systems to accelerate functional analysis of transcription factors using chimeric repressor gene-silencing technology (CRES-T). Plant Biotechnol 28: 201-210

Oshima Y, Shikata M, Koyama T, Ohtsubo N, Mitsuda N, OhmeTakagi M (2013a) MIXTA-like transcription factors and WAX INDUCER1/SHINE1 coordinately regulate cuticle development in Arabidopsis and Torenia fournieri. Plant Cell 25: 1609-1624

Panikashvili D, Savaldi-Goldstein S, Mandel T, Yifhar T, Franke RB, Höfer R, Schreiber L, Chory J, Aharoni A (2007) The Arabidopsis DESPERADO/atWBC11 transporter is required for cutin and wax secretion. Plant Physiol 145: 1345-1360

Pighin JA, Zheng H, Balakshin LJ, Goodman IP, Western TL, Jetter R, Kunst L, Samuels AL (2004) Plant cuticular lipid export requires an $\mathrm{ABC}$ transporter. Science 306: 702-704

Whitney HM, Kolle M, Andrew P, Chittka L, Steiner U, Glover BJ (2009) Floral iridescence, produced by diffractive optics, acts as a cue for animal pollinators. Science 323: 130-133

Winter D, Vinegar B, Nahal H, Ammar R, Wilson GV, Provart NJ (2007) An "Electronic Fluorescent Pictograph" browser for exploring and analyzing large-scale biological data sets. PLoS ONE 2: e718

Yang F, Mitra P, Zhang L, Prak L, Verhertbruggen Y, Kim JS, Sun L, Zheng K, Tang K, Auer M, et al. (2013) Engineering secondary cell wall deposition in plants. Plant Biotechnol J 11:325-335

Yang J, Isabel Ordiz MI, Jaworski JG, Beachy RN (2011) Induced accumulation of cuticular waxes enhances drought tolerance in Arabidopsis by changes in development of stomata. Plant Physiol Biochem 49: 1448-1455

Zhu L, Guo J, Zhu J, Zhou C (2014) Enhanced expression of EsWAX1 improves drought tolerance with increased accumulation of cuticular wax and ascorbic acid in transgenic Arabidopsis. Plant Physiol Biochem 75: 24-35 